\title{
Delayed Spontaneous Pneumothorax Presentation in Covid-19: Case Series
}

\section{and Review}

\section{Cara Owens | Hussain Amin | Samir Alwan | Indu Mitra*}

*Correspondence: Indu Mitra

Address: Chelsea and Westminster Hospital NHS Trust, London, UK

e-mail $\bowtie$ : indu_mitra@hotmail.com

Received: 20 October 2020; Accepted: 03 November 2020

Copyright: (C) 2020 Owens C. This is an open-access article distributed under the terms of the Creative Commons Attribution License, which permits unrestricted use, distribution, and reproduction in any medium, provided that the original work is properly cited.

\section{ABSTRACT}

Background: Since the presentation of the 2019 novel coronavirus (2019-nCoV), there have been several published papers outlining the clinical and radiological course of the disease. However there is minimal data regarding delayed complications.

Methods: In this retrospective, single-centre study, we identify patients who have presented with spontaneous pneumothorax, several weeks after their initial symptoms. All these cases are without history of recent invasive ventilation.

Findings: To date, we have four cases of spontaneous pneumothorax, presenting several weeks after initial symptoms of Covid-19. All these cases are male, with ages ranging from 40 to 90 years old. Only one had known interstitial lung disease, the others had normal previous lung imaging.

Interpretation: Current literature appears to be centred on the acute features of Covid-19. There is little data available regarding the sub-acute presentation of this disease. To our knowledge, there have only been a handful of reported cases of spontaneous pneumothorax, with the majority of papers reporting no cases. Our small case series highlights that the risk of spontaneous pneumothorax may be under-reported. We discuss the current known pathophysiological mechanisms of Covid-19 and discuss the potential delayed complications of this disease.

Keywords: Pneumothorax, Covid-19, SARS CoV-2, Thoracic Computed Tomography

\section{Introduction}

Since the reported cases from Wuhan on December 31, 2019, severe acute respiratory syndrome coronavirus 2 (SARS CoV-2) has fast become a pandemic, with over 4 million global cases and the first reported case in the UK on January 31, 2020.2 During this outbreak, thoracic computed tomography (CT) has played a pivotal role in disease management (Li Y et al., 2020) as well as evaluation of patients with suspected COVID-19 infection (with or without a positive RT-PCR result) (Bernheim et al., 2020; Huang et al., 2020). The clinical features (Chen et al., 2020) and radiological appearances on CT have been widely reported (Xu et al., 2020; Pan et al., 2020; Ding et al., 2020) with the most consistently 
reported CT feature of COVID-19 being ground glass opacification (often bilateral, peripheral or posterior) and predominantly located in lower lobes (Salehi et al., 2020). Approximately two weeks after onset (Pan et al., 2020), patients may develop confluent consolidations. It is about two to three weeks into the disease process, that CT may show bronchial wall and interlobular septal thickening. These features are found to be consistent with the archetypal response to acute lung injury (Xu XW et al., 2020). There are, however several less commonly reported findings, such as pleural effusion, pericardial effusion, lymphadenopathy and cavitation (Bernheim et al., 2020; Salehi et al., 2020). Another rare finding is pneumothorax which is believed to have a prevalence of $1 \%$ in patients with COVID-19 (Chen et al., 2020).

Development of a pneumothorax and/or pneumomediastinum, are recognised sequelae of mechanical ventilation in patients with underlying lung diseases (Hsu and Sun, 2014) and acute respiratory distress syndrome (Weg et al., 1998). It follows that patients with COVID-19 receiving noninvasive or invasive ventilation are at risk for these complications. However, there have been recently reported cases of secondary spontaneous pneumothorax in non-ventilated COVID-19 positive patients (Sun et al., 2020; Liu et al., 2020; Wang W et al., 2020; Rohailla et al., 2020; Kolani et al., 2020). It is vital that this rare complication is recognised and managed, both in ambulatory patients and in those destined for intensive treatment. In this retrospective case series we discuss four, non-ventilated, male patients with confirmed COVID-19, who developed pneumothoraces during the mid-late course of their active infection.

\section{Cases}

Case 1 is a 90 year old man recently returned from India woke suddenly at home at 4 am with sharp, left sided chest pain. On admission to hospital he did not require any oxygen therapy; COVID-19 RT-PCR was positive. Past medical history included poorly controlled type II diabetes, hypertension, chronic kidney disease, peripheral vascular disease, coronary artery disease, prostate cancer and mild, asymptomatic lung fibrosis (usual interstitial pneumonia (UIP) pattern was evident on a CT chest from 2015).

A chest radiograph showed bilateral patchy airspace opacification with reticulation at the bases (Fig. 1). Typical features of COVID-19 infection were evident on a CT pulmonary angiogram (CTPA) with bilateral, posterior, subpleural ground-glass opacification and scattered foci of interlobular septal thickening (Fig 2). In addition, there was a shallow left sided pneumothorax. No pulmonary embolus was detected. The patient was managed conservatively and discharged on day two with a plan for follow-up chest radiograph in two weeks. 


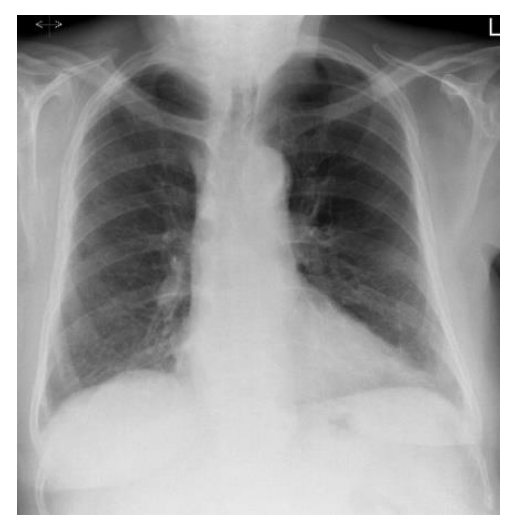

Figure 1: Chest radiograph of a 90 year old man with confirmed COVID-19 infection demonstrating bilateral, airspace opacification and stable basal reticulation

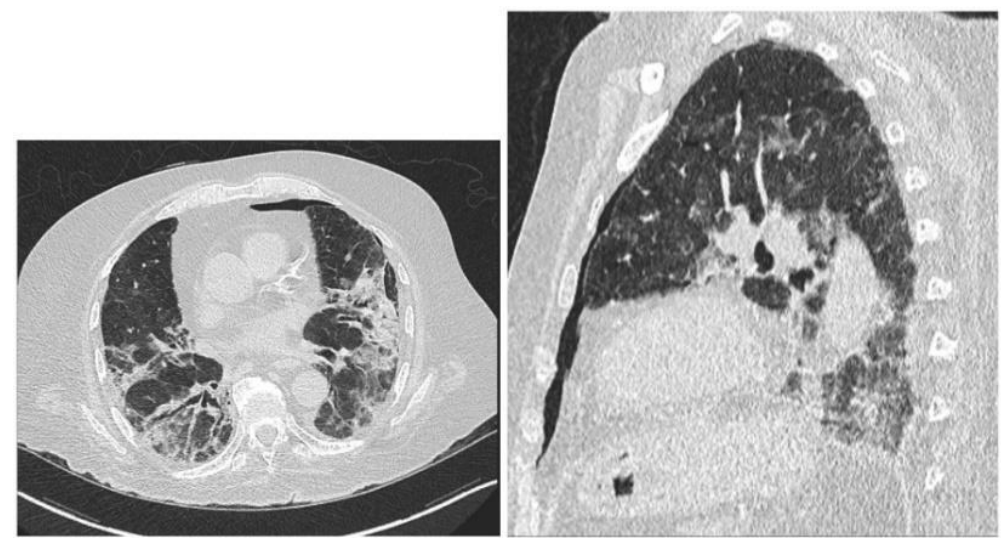

Figure 2: Axial (a) and sagittal (b) CT images of a 90 year old man with confirmed COVID-19 infection demonstrating bilateral, posterior, subpleural ground-glass opacification and scattered foci of interlobular septal thickening as well as a shallow left sided pneumothorax

Case 2 is a 40 year old man with confirmed COVID-19 infection was admitted in the early hours with acute, sharp, right sided chest pain. He had a three week history of cough, fever and chest pain and had just been discharged from hospital on the previous evening. He was saturating at 96\% on room air on admission. The patient had a medical history of bicuspid aortic valve with severe aortic regurgitation and was also obese. He had no underlying lung condition.

A chest radiograph on admission demonstrated bilateral airspace infiltrates (improved from previous chest radiograph) plus a new right midzone density suggestive for an encysted effusion (Fig. 3). A CTPA confirmed improved bilateral, patchy, peripheral ground glass opacification (Fig. 4). In addition there was a new encysted gas/fluid collection adjacent to the right oblique fissure consistent with a hydropneumothorax. A further, small right-sided hydropneumothorax had also developed. No pulmonary embolus was detected. The patient was treated conservatively and discharged on day three. The patient re-presented three times over the following month with ongoing shortness of breath. The hydropneumothoraces remained stable in appearance. 

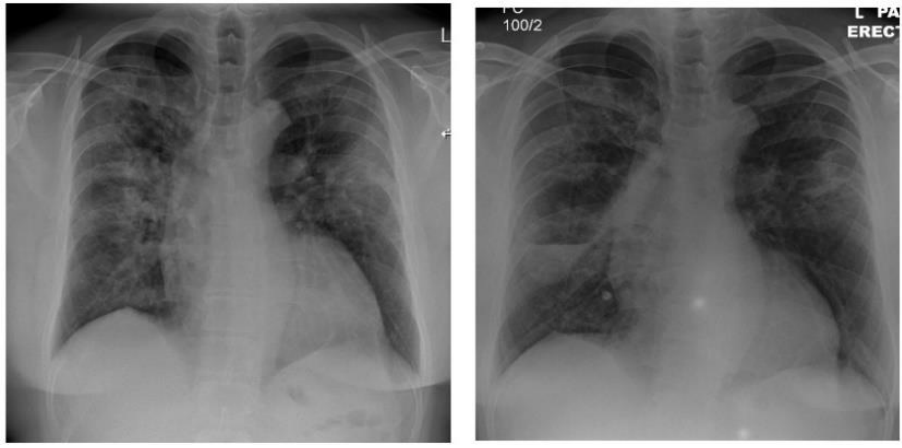

Figure 3: Chest radiographs of a 40 year old man with confirmed COVID-19 infection showing bilateral airspace infiltrates (a) which have improved on subsequent imaging (b). A new, encysted right pleural effusion is evident on the second radiograph

(b)
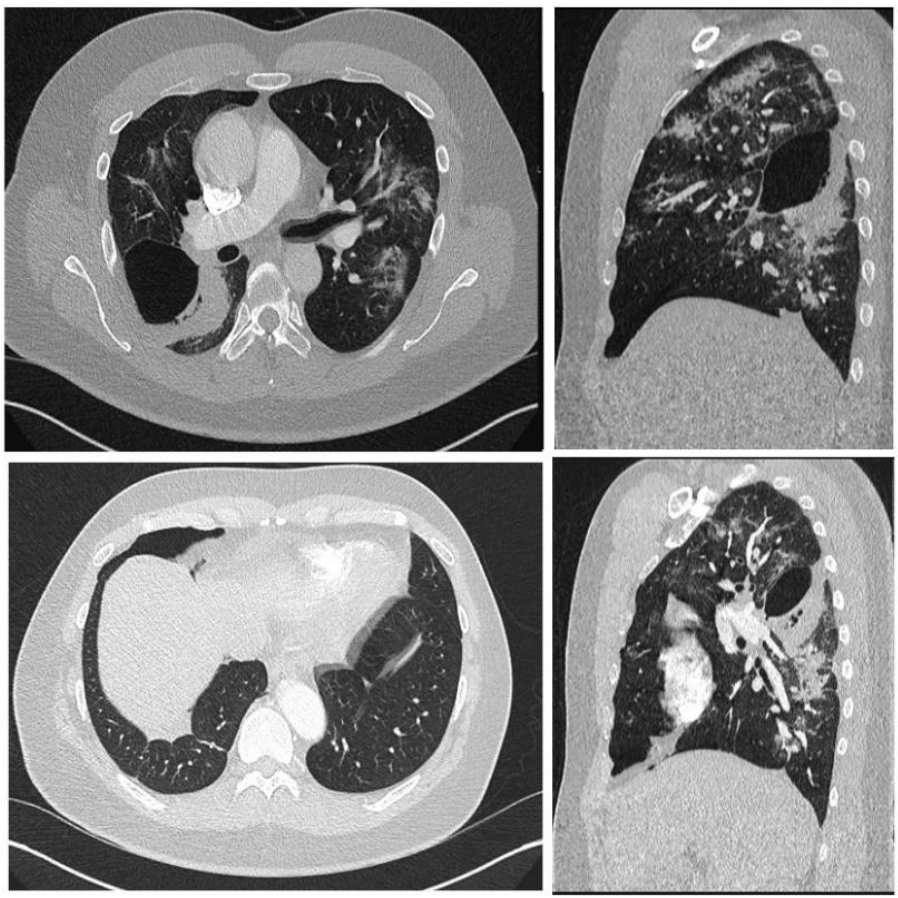

Figure 4: CTPA of a 40 year old man with confirmed COVID-19 infection. Axial (a) and sagittal (b) images show a right hydropneumothorax abutting the right oblique fissure on the background of bilateral multi-focal, groundglass opacification.

Axial (c) and sagittal (d) images taken at a different level show a further, smaller right basal hydropneumothorax $\left({ }^{*}\right)$

Case 3 is a 75 year old man with confirmed COVID-19 infection presented with chest pain and haemoptysis. He had recently been in hospital for 11 days for persistent shortness of breath (no NIV or intubation during the admission). He had a background medical history of COPD (GOLD stage 3; FEV1 $39 \%$ ) requiring home oxygen and mild obstructive sleep apnoea.

Chest radiograph on admission showed bilateral atelectasis plus an air-fluid level at the left costophrenic angle (Fig. 5). A CTPA demonstrated interval improvement of bilateral ground glass opacification from imaging 18 days previously but the development of several areas of more confluent 
consolidation (Fig. 6). In addition, a new small-moderate left hydropneumothorax had developed. No pulmonary embolus was detected. The patient was treated conservatively and discharged on the same day.

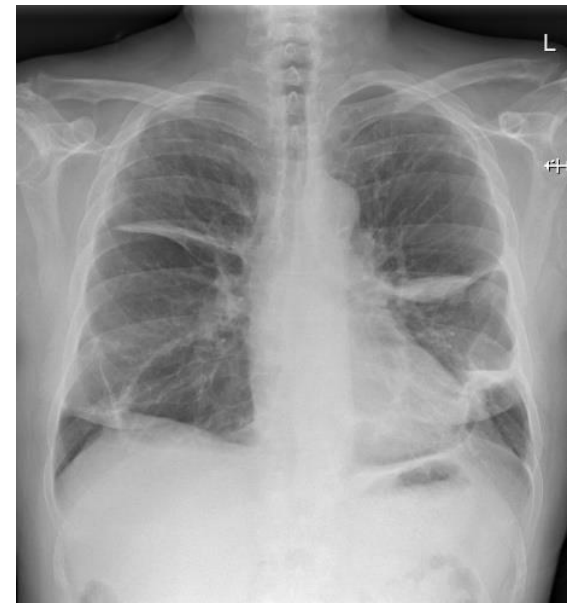

Figure 5: Chest radiograph of a 75 year old man with confirmed COVID-19 infection demonstrating multiple, bilateral foci of atelectasis plus an air-fluid level at the left base
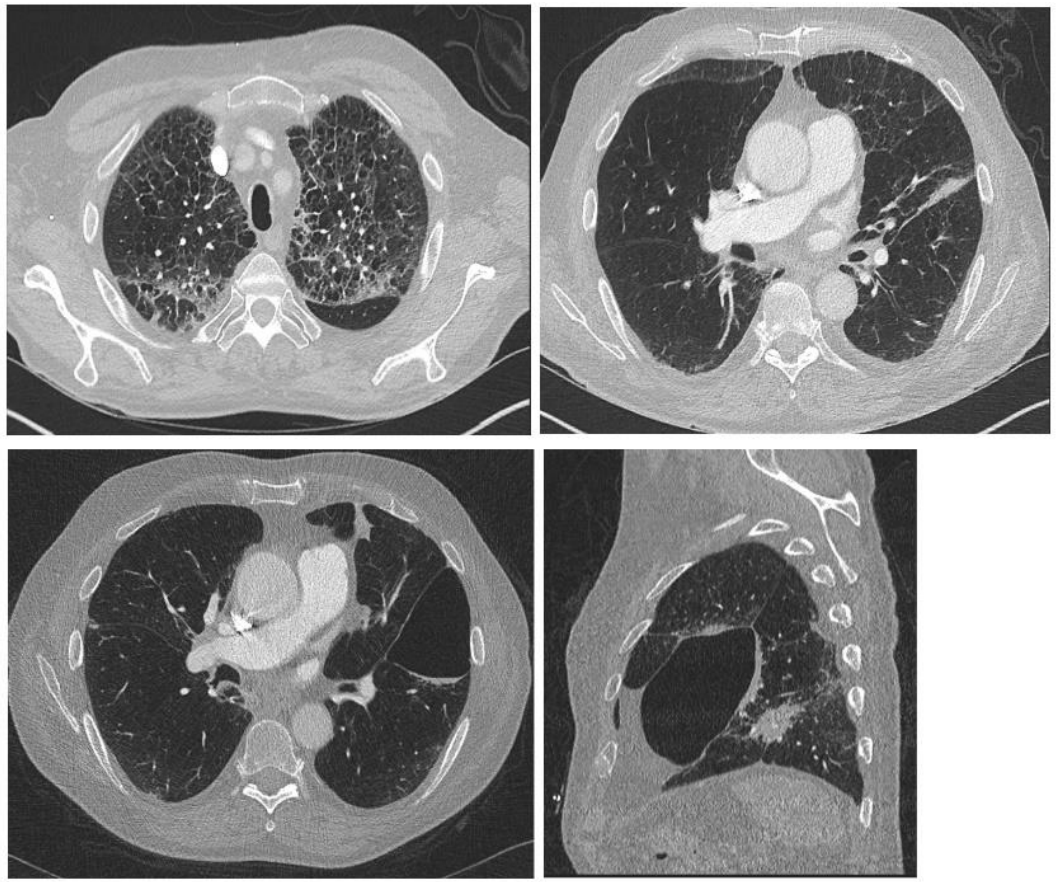

Figure 6: CTPA of a 75 year old man with confirmed COVID-19 infection on initial presentation (a, b) and 18 days later (c, d).

Bilateral ground glass opacification is present on a background of moderate-severe upper lobe predominant centrilobular emphysema $(\mathrm{a}, \mathrm{b})$. On the follow up images, the ground glass component has reduced with evidence of confluent consolidation/organising pneumonia. A new small-moderate left hydro pneumothorax has now developed

Case 4 is an 80-year-old man with confirmed COVID-19 infection presented with a two day history of sudden onset left-sided chest pain and shortness of breath following an intense bout of coughing, 40 
days following initial symptoms. Past medical history included ischaemic heart disease, hypertension, chronic renal failure (stage 3) and recently diagnosed COPD. On initial medical assessment he was short of breath with oxygen saturations in the low 90s, improving to $95 \%$ on $2-3 \mathrm{~L}$ via nasal cannula.

Chest radiograph on admission demonstrated a new left-sided pneumothorax (Fig. 7). CT chest confirmed a large left-sided pneumothorax with mild mediastinal shift to the right plus classic COVID-19 lung changes with superimposed bilateral lower lobe consolidation (Fig. 8).

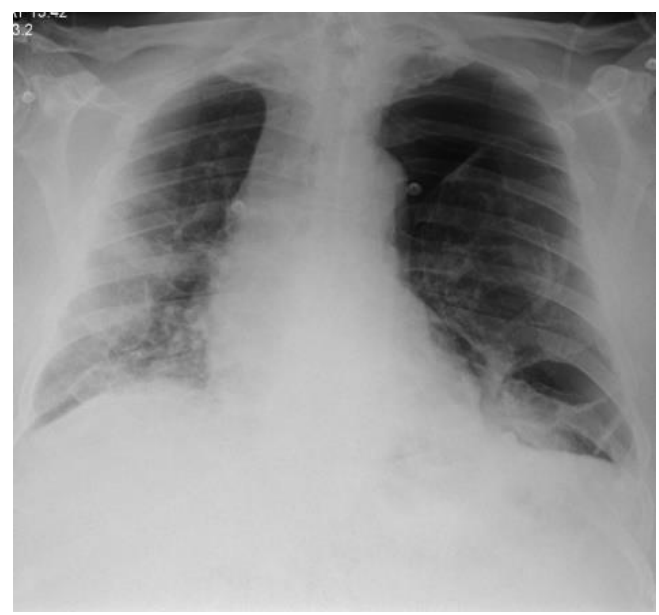

Figure 7: Chest radiograph of an 80 year old man with confirmed COVID-19 infection demonstrating a left sided pneumothorax plus bilateral peripheral infiltrates

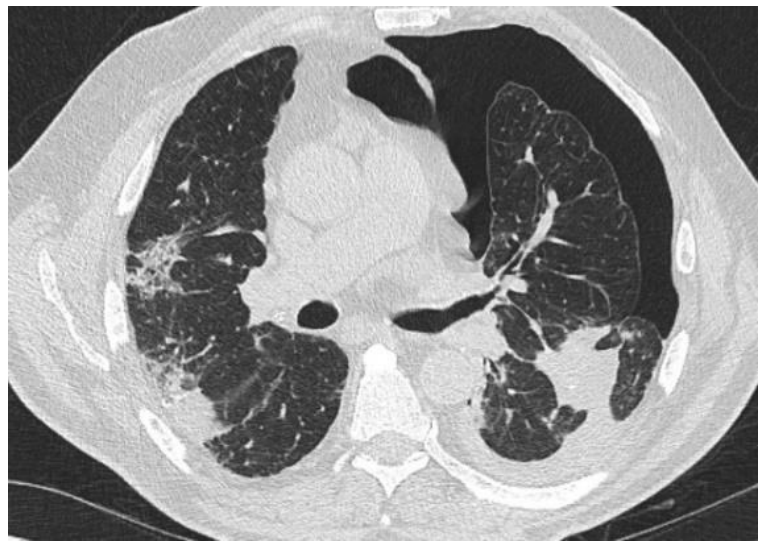

Figure 8: Axial CT image of an 80 year old man with confirmed COVID-19 infection demonstrating a moderate left pneumothorax causing rightward mediastinal shift on a background of bilateral, peripheral ground glass and confluent consolidation

The patient was managed with a left-sided percutaneous chest drain and intravenous antibiotics to cover for secondary bacterial infection. Microbiological test revealed negative blood cultures and pleural fluid and two repeat COVID-19 swabs were negative. The patient made significant clinical improvement following this management and was discharged on day three with four weeks of oral antibiotics. 


\section{Discussion}

All our cases had real-time RT-PCR positive tests for SARS-CoV-2 and were male. Male prevalence in Covid-19 has previously been noted (Chen et al., 2020). There is, however, a published case report of spontaneous pneumomediastinum in a 23 year old female, implying that this phenomenon, although prevalent in males is not exclusive.

The age range of our cases varied from 40 to 90 years old. Some of the patients had co-morbidites, such as, diabetes mellitus, hypertension, chronic renal failure, cardiovascular disease and obesity; these have previously been reported in 'high risk' groups (Wang D et al., 2020; Michalakis and Ilias, 2020; Rothan and Byrareddy, 2020). Only Case 1 had a history of previous interstitial lung disease with minor existing basal fibrosis in keeping with mild usual interstitial pneumonia. The others, apart from mild chronic obstructive airways disease and obstructive sleep apnoea, had normal appearing lungs on previous imaging. It can only be presumed that the presentation of spontaneous pneumothorax is associated with the acute lung changes from SARS-CoV-2 infection.

The terms acute lung injury (ALI) and acute respiratory distress syndrome (ARDS) are not new, and have been revised over the years to include clinical and radiological features (Desai, 2002). Following on from the 2002 severe acute respiratory syndrome (SARS CoV) (Vijaynand et al., 2004), much work has been undertaken to understand the pathophysiological progression in Covid-19 (Kowalik et al., 2020), from acute lung injury (ALI) to the more severe acute respiratory distress syndrome (ARDS) (Gralinski et al., 2013). Both of these processes are stereotyped with the histological hallmark of DIFFUSE alveolar damage (Desai, 2002). There are three main principal phases described: the exudative, proliferative and chronic phases. In the first exudative phase, inflammatory exudates move into the interstitium, proteinaceous fluid floods the alveoli, the alveoli collapse and hyaline membranes form. This is followed by the proliferative phase where fibroblasts and type II pneumocytes proliferate and there may be persistent alveolar consolidation due to inflammation and haemorrhage. In the final chronic phase, there is proliferation of type I pneumocytes and collagen deposition (Desai, 2002). In some patients this can lead to chronic interstitial fibrosis. It is true that this fibrosis can occur rapidly and early in ALI/ARDS.

These phases are reflected in the CT imaging features of COVID-19, which can be categorised8 into four temporal stages. Stage 1 is usually less than four days from onset of symptoms, and is characterised by groundglass opacification, which likely represents pulmonary oedema/haemorrhage and hyaline membrane formation (Pan et al., 2020; Ye Z et al., 2020). As the disease progresses in stage 2 (5-8 days), crazy-paving develops reflecting alveolar oedema and the interstitial inflammation of acute lung injury. 
Confluent consolidation is the main CT imaging feature of stage 3 (9-13 days) and is probably caused by the accumulation of fibromyxoid exudates in alveoli. Stage 4 occurs 14 days after initial symptoms and describes resolution of consolidation (Pan et al., 2020). It is this close correlation between imaging features and pathological change, that has proven CT imaging to be valuable in evaluating severity and extent of disease (Bernheim et al., 2020). In our case series, all the patients were in the late stages of their Covid-19 infection, given their chest CTs showing a combination of residual ground glass opacification, confluent consolidation and crazy-paving.

In this case series, we have described the spontaneous presentation of the air filled intrathoracic foci as pneumothoraces. According to the Fleischner Society (Hansell et al., 2008), the definition of a pneumothorax is the presence of gas in the pleural space. Cases 1 and 4 are classical appearances for pneumothoraces. In the other two cases, it could be argued that these are cavities, pneumatoceles or air filled cysts. Technically in these cases, the air filled spaces are adjacent to fissures and presumably within the pleural space rather than being completely surrounded by lung, hence the description of localised pneumothorax.

Our case series highlights that the prevalence of spontaneous pneumothorax in Covid-19 may be higher than initially expected. This would follow suit, given that in the 2002 SARS epidemic, 6 in 365 patients suffered from spontaneous pneumothorax (Sihoe et al., 2004). The published image of the localised pneumothorax described then, is very similar to those in our cases 2 and 4 . It could be that there is a similarity and that the resultant acute lung injury-associated early fibrosis in Covid-19 patients (Ye Z et al., 2020) may cause traction with subsequent air leak. Another theory is that pneumothorax develops secondary to subpleural bulla rupture (Light, 1993) or cyst formation (Chen et al., 2020). These are thought to develop due to ischaemic parenchymal damage, lung fibrosis, low lung compliance and inflammatory exudate in the airway causing bullae formation.

It is difficult to know the true prevalence of these air filled spaces in Covid-19 patients, with varying descriptions and accounts. One paper reported no pneumothorax in non ventilated Covid-19 patients (Chen et al., 2020), and another one case in 99 patients (Kong and Prachi, 2020). There is one report of a small cavitation (Shi et al., 2020) and another paper stating no cavitations (Wu J et al., 2020). Another paper has reported $1.5 \%$ pneumatocele presentation, with no pulmonary cavities and a $2.3 \%$ rate of pleural effusion. Of the five published case reports published (Sun et al., 2020; Liu et al., 2020; Wang W et al., 2020; Rohailla et al., 2020; Kolani et al., 2020), four were male and one female. Age range varied from 23 to 62 years old. The time of presentation was earliest 3 days to latest 20 days from onset of symptoms. Two patients had received non invasive ventilation. Cyst formation was noted in one of the cases prior to development of pneumothorax. 
This article highlights that the development of spontaneous pneumothorax in Covid-19 patients of varying ages, with and without preceding co-morbidiities and the majority presenting later on in the disease process. This has implications for short and long term patient management. Considerations in the acute setting include the feasibility and success rate of drainage or pleurodesis, implications for assisted ventilation and the risk of life threatening tension pneumothorax development. Longer term consideration must be given to the effect on patient lung function and the need for specialist respiratory monitoring with a likely increased demand on imaging.

\section{Learning Points}

- The incidence of pneumothorax in non-ventilated patients with COVID-19 infection may be underreported.

- Early fibrosis, traction and air-leak, cyst formation and intense coughing may result in pneumothorax development in patients with COVID-19 infection.

- Clinicians must consider pneumothorax development in ambulatory patients with COVID-19 infection especially if patients report new pleuritic chest pain or an exacerbation of their breathlessness.

- Spontaneous pneumothorax development will impact on the initial management of patients with COVID-19 infection and has implications for future pulmonary health.

- Loculated hydropneumothoraces pose particular challenges in terms of clinical management and future pulmonary health.

\section{References}

Bernheim A, Mei X, Huang M, Yang, Y, Fayad, Z, Zhang, N, et al. Chest CT Findings in Coronavirus Disease-19 (COVID-19): Relationship to Duration of Infection. Radiology 2020; 80: 1-8.

Chen N, Zhou M, Dong X, Qu J, Gong F, Han Y, Qiu Y, Wang J, Liu Y, Wei Y, Yu T. Epidemiological and clinical characteristics of 99 cases of 2019 novel coronavirus pneumonia in Wuhan, China: a descriptive study. Lancet 2020; 395: 507513 .

Chen N, Zhou M, Dong X, Qu J, Gong F, Han Y, Qiu Y, Wang J, Liu Y, Wei Y, Yu T. Epidemiological and clinical characteristics of 99 cases of 2019 novel coronavirus pneumonia in Wuhan, China: a descriptive study. Lancet 2020; 395 : 507513.

Desai SR. Acute respiratory distress syndrome: Imaging of the injured lung. Clin Radiol 2002; 57: 8-17.

Ding X, Xu J, Zhou J, Long Q. Chest CT findings of Covid-19 pneumonia by duration of symptoms. Eur J Radiol 2020; 127 : 109009.

Gralinski LE, Bankhead A, Jeng S, Menachery VD, Proll S, Belisle SE, Matzke M, Webb-Robertson BJ, Luna ML, Shukla AK, Ferris MT. Mechanisms of Severe Acute Respiratory Syndrome Coronavirus-Induced Acute Lung Injury. mBio 4: e00271-13. 
Hansell DM, Bankier AA, MacMahon H, McLoud TC, Muller NL, Remy J. Fleischner Society: Glossary of Terms for Thoracic Imaging. Radiology 2008; 246: 697- 722.

Hsu CW and Sun SF. Iatrogenic pneumothorax related to mechanical ventilation. World J Crit Care Med 2014; 3: 8-14.

Huang P, Liu T, Huang L, Liu H, Lei M, Xu W, Hu X, Chen J, Liu B. Use of Chest CT in Combination with Negative RT-PCR Assay for the 2019 Novel Coronavirus but High Clinical Suspicion. Radiology 2020; 295: 22-23.

Kolani S, Nawfal H, Haloua M, Lamrani YA, Boubbou M, Serraj M, Aamara B, et al. Spontaneous pneumomediastinum occurring in the SARS-COV-2 infection. IDCases 2020; 11: e00806. e200028.

Kong W, Prachi P. Chest Imaging Appearances of COVID-19 Infection. Radiology: Cardiothoracic Imaging 2020; 12 :

Kowalik MM, Trzonkowski P, Łasińska-Kowara M, Mital A, Smiatacz T, Jaguszewski M. Covid-19 - toward a comprehensive understanding of the disease. Cardiology Journal 2020; 27: 99-114.

Li Y and Xia L. Coronavirus Disease 2019 (COVID-19): Role of Chest CT in Diagnosis and Management. AJR Am J Roentgenol 2020; 4: 1-7.

Light RW. Management of spontaneous pneumothorax. Am Rev Respir Dis 1993: 148: 245-248.

Liu K, Zeng Y, Xie P, Ye X, Xu G, Liu J, Wang H, Qian J. COVID-19 with cystic features on computed tomography. Medicine (Baltimore) 2020; 18: 19-21.

Michalakis K and Ilias I. SARS-CoV-2 infection and obesity: Common inflammatory and metabolic aspects. Diabetes \& Metabolic Syndrome: Clinical Research \& Reviews 2020; pp: 469-471.

Pan F, Ye T, Sun P, Gui S, Liang B, Li L, Zheng D, Wang J, Hesketh RL, Yang L, Zheng C. Time Course of Lung Changes On Chest CT During Recovery From 2019 Novel Coronavirus (COVID-19) Pneumonia. Radiology 2020; 200370.

Rohailla S, Ahmed N, Gough K. SARS-Cov-2 infection associated with spontaneous pneumothorax. CMAJ 2020; 192: E510.

Rothan HA and Byrareddy SN. The epidemiology and pathogenesis of coronavirus disease (COVID-19) outbreak. J of Automimmunity 2020; 109.

Salehi S, Abedi A, Balakrishnan S, Gholamrezanezhad A. Coronavirus Disease 2019 (COVID-19): A Systematic Review of Imaging Findings in 919 Patients. AJR Am J Roentgenol 2020; 14: 1-7.

Shi H, Han X, Jiang N, Cao Y, Alwalid O, Gu J, Fan Y, Zheng C. Radiological findings from 81 patients with Covid-19 pneumonia in Wuan, China: a descriptive study. Lancet Infect Dis 2020; 20: 425-434.

Sihoe AD, Wong RH, Lee AT, Lau LS, Leung NY, Law KI, Yim AP. Severe acute repiratory syndrome complicated by spontaneous pneumothorax. Chest 2004; 125: 2345-2351.

Sun R, Liu H, Wang X. Mediastinal Emphysema, Giant Bulla, and Pneumothorax Developed during the Course of COVID19 Pneumonia. Korean J Radiol 2020; 21: 541-544.

Vijaynand P, Wilkins E, Woodhead M. Severe acute respiratory syndrome (SARS): a review. Clin Med 2004; 4: 152-160.

Wang D, Hu B, Hu C, Zhu F, Liu X, Zhang J, Wang B, Xiang H, Cheng Z, Xiong Y, Zhao Y. Clinical characteristics of 138 hospitalised patients with 2019 Novel Coronavirus - infected Pneumonia In Wuhan, China. JAMA 2020; 323: 1061 -1069.

Wang W, Gao R, Zheung Y, Jiang L. Covid-19 with spontaneous pneumothorax, pneumomediastinum and subcutaneous 
emphysema. J of Travel Medicine. 27: taaa062.

Weg JG, Anzueto A, Balk RA, Wiedemann HP, Pattishall EN, Schork MA, Wagner LA. The relation of pneumothorax and other air leaks to mortality in the acute respiratory distress syndrome The New England Journal of Medicine 1998; 338: 341346.

Wu J, Feng LC, Xian XY, Qiang J, Zhang J. Novel coronavirus pneumonia (COVID-19) CT distribution and sign features. Zhonghua Jie He He Hu Xi Za Zhi 2020; 43: 321-326.

Xu X, Yu C, Qu J, Zhang L, Jiang S, Huang D, Chen B, Zhang Z, Guan W, Ling Z, Jiang R. Imaging and clinical features of patients with 2019 novel coronavirus SARS-CoV-2. European journal of nuclear medicine and molecular imaging 2020; 28 : 1-6.

Xu XW, Wu XX, Jiang XG, Xu KJ, Ying LJ, Ma CL, Li SB, Wang HY, Zhang S, Gao HN, Sheng JF. Clinical findings in a group of patients infected with the 2019 novel coronavirus (SARS-Cov-2) outside of Wuhan, China: Retrospective case series. BMJ 2020; 368: 1-7.

Ye Z, Zhang Y, Wang Y, Huang Z, Song B. Chest CT manifestations of new coronavirus disease 2019 (COVID-19): a pictorial review. Eur Radiol 2020; 19: 1-9. 